\title{
A técnica da resolução das equações Relativas à Interpolação da Lei de Mitscherlich pelo Método dos Quadrados Mínimos
}

IZAIAS RANGEL NOGUEIRA

Assistente substituto da $16 a$. cadeira da E. S. A.

"Luiz de Queiroz"

Conforme mostraram Pimentel Gomes e Malavolta, a interpolação dạ equação de Mitscherlich

$$
y=A\left[1-10^{-c(x+b)}\right]
$$

pelo método dos quadros mínimos c nduz á equação em $\mathrm{c}$ :

(I) $\left|\begin{array}{lcc}\Sigma_{\mathrm{yi}} & \mathrm{n} & \Sigma_{10}-\mathrm{cxi} \\ \Sigma_{\mathrm{i}} \mathrm{y}_{\mathrm{i}} 10^{-\mathrm{cx}} & \Sigma_{\mathrm{i}} 10^{-\mathrm{cx}} & \Sigma 10^{-2 \mathrm{cxi}} \cdot \mathrm{x}_{\mathrm{i}} \\ \Sigma_{\mathrm{y}_{\mathrm{i}} 10^{-c x_{i}}} & \Sigma 10^{-\mathrm{cx}} & \Sigma 10^{-2 \mathrm{cx}} \mathrm{i}\end{array}\right|=0$

cuja resolução se consegue mais fàcilmente tomando: 
$x i=$ q.mi $\quad(i=1,2, \ldots \ldots, n)$, sendo mi um número inteiro, e fazendo :

$$
\mathrm{Z}=10^{-\mathrm{cq}} \text { obtem-se assim a equação al- }
$$

(II) $\left|\begin{array}{lcc}\Sigma \mathbf{y}_{\mathrm{i}} & \mathrm{n} & \Sigma \mathrm{z}_{\mathrm{i}}^{\mathrm{m}_{\mathrm{i}}} \\ \Sigma \mathrm{x}_{\mathrm{i}} \mathrm{y}_{\mathrm{i}} \mathrm{z}^{\mathrm{m}_{\mathrm{i}}} & \Sigma \mathrm{x}_{\mathrm{i}} \mathrm{z}_{\mathrm{i}}^{\mathrm{m}_{\mathrm{i}}} & \Sigma \mathrm{z}^{2 \mathrm{~m}_{\mathrm{i}}} \cdot \mathrm{x}_{\mathrm{i}} \\ \Sigma \mathrm{y}_{\mathrm{i}} \mathrm{z}^{\mathrm{m}_{\mathrm{i}}} & \Sigma \mathrm{z}^{\mathrm{m}_{\mathrm{i}}} & \Sigma \mathrm{z}^{2 \mathrm{~m}_{\mathrm{i}}}\end{array}\right|=0$

cujo desenvolvimento pode ser feito por qualquer dos métodos conhecidos.

Achamos preferivel, porém, desenvolver o determinante segundo os elementos da primeira coluna, $\theta$ isso pelos motivos seguintes :

1) Com excessão desta 1a. coluna todos demais elementos do determinante não contêm valores de $\mathrm{y}$.

Isto significa que se as mesmas doses de fertilisantes foram usadas para diversas experiências, aquêles elementos têm o mesmo valor para tôdas elas, de sorte que os complementos algébricos dos elementos da 1a. coluna são sempre os mesmos.

2) Observando o que acontece ao determinante quando $\mathbf{Z}=1$, temos :

(III) $\Delta\left({ }_{1}\right)=\left|\begin{array}{ccc}\Sigma \mathbf{y}_{\mathbf{i}} & \mathbf{n} & \mathbf{n} \\ \Sigma \mathbf{x}_{\mathbf{i}} \mathbf{y}_{\mathbf{i}} & \Sigma \mathbf{x}_{\mathbf{i}} & \Sigma \mathbf{x}_{\mathbf{i}} \\ \Sigma \mathbf{y}_{\mathbf{i}} & \mathbf{n} & \mathbf{n}\end{array}\right|$

concluimos que os menores correspondentes à 1a. coluna são nulos (pois tem cada um dêles 2 colunas iguais).

Se êles são nulos, os polinômios dêles oriundos, por desenvolvimento, se anulam para $Z=1$, isto é, a soma de seus coeficientes deve ser nula. Dai se conclui que são divisiveis por $(\mathrm{Z}-1)$. 
Esta propriedade nos auxilia muito no desenvolvimento, pois que nos permite tirar a prova dos nossos cálculos, simplesmente somando os coeficientes de $\mathrm{Z}$ em cada polinômio.

Éstes devem, como vimos acima, ter soma nula.

O 1o. desenvolvimento, segundo a 1a. coluna, conduz então à equação :

$$
P_{1}(Z) \Sigma_{y_{i}}+P_{2}(Z) \Sigma_{x_{1} y_{i}} Z^{m i}+P_{3}(Z) \Sigma_{y_{i} Z} Z^{m i}=0
$$

* onde $P_{1}(Z), P_{2}(Z)$ e $P_{3}(Z)$ são nulos para $Z=1$

Efetuando agora êsses produtos, chegamos ao polinômio P (Z) de gráu geralmente elevado.

Este também para $\mathrm{Z}$ igual a 1 , deve dar uma soma de coeficiente nula, pois (I) deve anular-se para êsse valor de $Z$.

Mas uma vez portanto, podemos tirar a prova dos nossos cálculos.

Vamos a seguir determinar o valor da raiz $Z$ da equação (I), que como se sabe (Pimentel Gomes e Malavolta, 1949, pg. 15), deve estar compreendida entre 0 e 1.

A localização da raiz de uma equação de grau elevado é feita por tentativa, localizando-se primeiramente o intervalo onde ela se encontra.

No nosso caso, como acima referimos, ela já está localizada. Procuraremos então no intervalo citado, o valor mais aproximado da raiz, seguindo para tal o processo de Briot-Ruffini (um exemplo numérico esclareceria bem a marcha a seguir).

Uma vez localizada a raiz, calculamos os outros parâmetros da equação de Mitscherlich, pelo processo já referido por Pimentel Gomes e Malavolta.

Como um exemplo, apresentamos um trabalho por nós pesquizados, em dados de calagem de trigo, realizado em Ponta Grossa, em 1947.

As doses de cal utilizadas foram de 2, 4, 6 e 8 toneladas por $\mathrm{Ha}$, e as médias dos valores obtidos, 2378, 2658, 2724 e 2798 $\mathrm{Kg} / \mathrm{Ha}$, sendo que a testemunha deu $2.040 \mathrm{Kg}$.

Se tomarmos como unidade, para facilitar o cálculo, 2 toneladas/Ha, as doses de cal serão $0,1,2,3$ e 4.

Em resumo teremos

(*) Evidentemente os polinômios P1 (Z), P2 (Z) e P3 (Z) podem ser tabulados, trazendo êste fato grande vantagem para cálculo de $Z$ 


\begin{tabular}{c|c} 
Doses & Valores obtidos \\
0 & 2040 \\
1 & 2378 \\
2 & 2658 \\
3 & 2724 \\
4 & 2798 \\
\hline Soma total : & 12.598
\end{tabular}

Utilizando a equação (II) e desenvolvendo o determinante segundo os elementos da 1a. coluna obtemos :

$12.5988\left(1+\mathrm{Z}+3 \mathrm{Z}^{2}+3 \mathrm{Z}^{3}+\mathrm{Z}^{4}-\mathrm{Z}^{6}-3 \mathrm{Z}^{7}-3 \mathrm{Z}^{8}-\mathrm{Z}^{9}-\right.$ $-Z 10)-(2378+5316 Z+8172 Z 2+11.192 Z 3)(4-2 Z+$ $\left.2 Z 2-4 Z^{3}-4 Z^{5}+2 Z^{6}-2 Z^{7}+4 Z 8\right)+(2040+$

$\left.+2378 Z+2658 Z^{2}+2724 Z^{3}+2798 Z 4\right)\left(-1+2 Z-6 Z^{2}-\right.$ $-10 \mathrm{Z} 4+6 \mathrm{Z} 5-7 \mathrm{Z} 6+16 \mathrm{Z} 7)=0$

Os têrmos grifados dão, conforme podemos verificar, um valor nulo para $Z=1$.

Efetuando os produtos acima obtemos a equação final : $1096 \mathrm{Z} 10+346 \mathrm{Z} 9-444 \mathrm{Z} 8-6280 \mathrm{Z} 7+5298 \mathrm{Z} 6-2472 \mathrm{Z5}+$ $+6204 \mathrm{Z} 4-3426 \mathrm{Z}^{3}+840 \mathrm{Z}^{2}-2208 \mathrm{Z}+1046=0$

Esta equação também tem valor nulo para $\mathrm{Z}=1$.

Efetuaando a pesquisa da raiz pelo método de Briot-Ruffini, encontramos $\mathrm{Z}=0,5614$

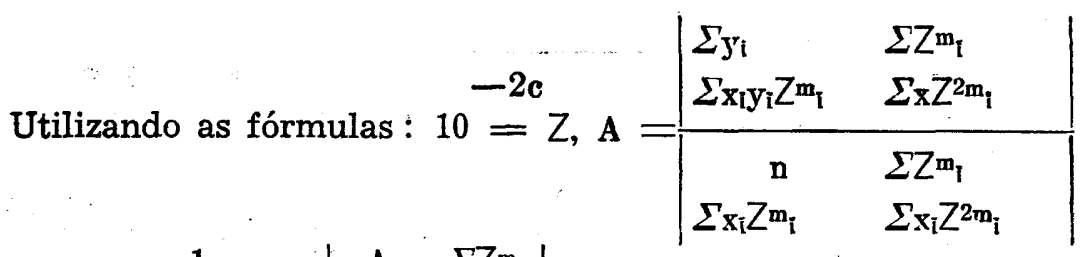
e b $=\frac{1}{\mathrm{c}} \log \left|\frac{\mathrm{A} \cdot \Sigma \mathrm{Z}_{\mathrm{i}}}{\mathrm{nA}-\Sigma \mathrm{y}_{\mathrm{i}}}\right|$

(Fórmulas estas indicadas no trabalho de Pimentel Gomes e Malavolta, pg 7), encontramos :

$c=0,12536 \quad A=2.887,3 \quad$ e $\quad b=4,22$ 
Substituindo êsses valores na equação $y=A\left[1-10^{-c(x+b)}\right]$ temos

$y=2.887,3\left[1-10^{-0,12536(x+4,22)}\right]$

Substituindo os valores de $x$ (doses de fertilisantes), nesta equação, encontramos os seguintes valores :

Em resumo:

\begin{tabular}{|c|c|c|}
\hline Valores & Observados & Valores esperados \\
\hline & $\begin{array}{l}2040 \\
2378 \\
2658 \\
2724 \\
2798\end{array}$ & $\begin{array}{c}2.033,3 \\
2.407,8 \\
2.618,1 \\
2.736,2 \\
2.802,5\end{array}$ \\
\hline mas tota & 12.598 & $12.597,9$ \\
\hline
\end{tabular}

BIBLIOGRAFIA CITADA

PIMENTEL Gomes Frederico e Euripedes MALAVOLTA

Considerações Matemáticas sôbre a Lei de Mitscherlich. Piracicaba 1949. 
